\title{
The thermal SZ power spectrum
}

\author{
Klaus Dolag ${ }^{1}$, Eiichiro Komatsu ${ }^{2}$ and Rashid Sunyaev ${ }^{2}$ \\ ${ }^{1}$ Universitäts-Sternwarte München, Scheinerstrasse 1, 81679 München, Germany \\ email: dolag@usm.uni-muenchen.de \\ Max-Planck-Institut für Astrophysik, Karl-Schwarzschild Str. 1, 85748 Garching, Germany
}

\begin{abstract}
The Magneticum Pathfinder (www.magneticum.org) cosmological, hydro-dynamical simulation $\left(896 h^{-1} \mathrm{Mpc}\right)^{3}$ follows in detail the thermal and chemical evolution of the ICM as well as the evolution of SMBHs and their associated feedback processes. We demonstrate that assuming cosmological parameters inferred from the CMB, the thermal SZ power spectrum as observed by PLANCK is well matched by the deep light-cones constructed from these cosmological simulations. The thermal SZ prediction from the full SZ maps are significantly exceeding previous templates at large $l$ (e.g., $l>1000)$ and therefore predict a significantly larger contribution to the signal at $l=3000$ compared to previous findings. The excess of positive values within the probability distribution of the thermal SZ signal within the simulated light-cone agrees with the one seen by PLANCK. This excess signal follows a power law shape with an index of roughly -3.2 . The bulk of the thermal SZ signal originates from clusters and groups which form between $z=0$ and $z \approx 2$ where at high redshift $(z>1)$ significant part of the signal originates from proto-cluster regions, which are not yet virialized. The simulation predicts a mean fluctuating Compton $\mathrm{Y}$ value of $1.18 \times 10^{-6}$, with a remaining contribution of almost $5 \times 10^{-7}$ when removing contribution from halos above a virial mass of $10^{13} M_{\odot} / h$.
\end{abstract}

Keywords. hydrodynamics, numerical, galaxy clusters, cosmic background radiation

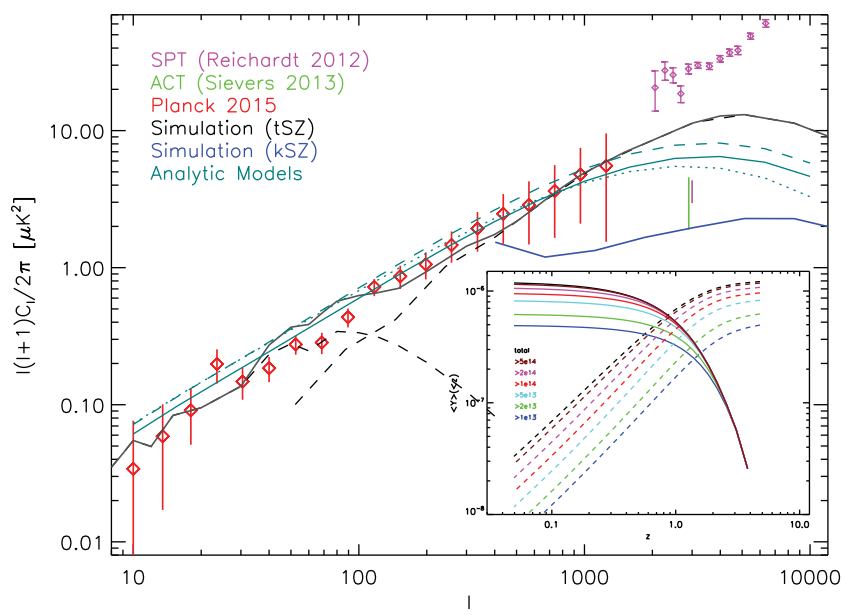

Figure 1. The observed angular power spectrum at $150 \mathrm{GHz}$ of the CMB from PLANCK (red data points) and SPT (pink data points, with only the primary signal subtracted). The black solid line shows the thermal SZ signal obtained from our simulation assuming CMB cosmology. The light blue solid line shows the analytic prediction assuming the mass function from Bocquet et al. (2015), while the dashed line uses the mass function from Tinker et al. (2008). The inlay shows the build up (solid lines) of the mean Compton Y parameter with declining redshift and the contribution of halos with different mass to it (color coded). The simulation predicts a mean Compton Y value of $1.18 \times 10^{-6}$, see Dolag, Komatsu \& Sunyaev (2015,arXiv:1509.05134). 